\title{
A LINK BETWEEN LEBESGUE CONSTANTS AND HERMITE-FEJÉR INTERPOLATION
}

\author{
S.J. GOODENOUGH
}

\begin{abstract}
A review of the development of estimates for Lebesgue constants associated with Lagrange interpolation on the one hand, and estimates for the rate of convergence of Hermite-Fejer interpolation on the other hand, provides a historical perspective for the following surprising, close link between these apparently diverse concepts. Denoting by $\Lambda_{n}(T)$ the Lebesgue constant of order $n$ and by $\Delta_{n}(T)$ the maximum intexpolation error for functions of class Lip 1 by Hermite-Fejér interpolation polynomials of degree not exceeding $2 n-1$, based on the zeros of the Chebyshev polynomial of the first kind, we discover that, for even values of $n$,

$$
\Lambda_{n}(T)=n \Delta_{n}(T) \text {. }
$$

Introduction
\end{abstract}

For $k=1,2,3, \ldots, n$, denote by

$$
x_{k}:=\cos \frac{(2 k-1) \pi}{2 n}
$$

the zeros of the Chebyshev polynomial of the first kind of degree $n$

$$
T_{n}(x):=\cos n \theta \text { where }-1 \leqslant x:=\cos \theta \leqslant 1 \text {. }
$$

Furthermore, denote by $T$ the infinite triangular matrix whose $n$th row consists of the points $x_{1}, x_{2}, x_{3}, \ldots, x_{n}$ given by (1). Henceforth, $T$ will be termed the Chebyshev matrix.

Received 4 June 1985.

Copyright Clearance Centre, Inc. Serial-fee code: 0004-9727/86 $\$ A 2.00+0.00$. 
The Lebesgue constant, $\Lambda_{n}(T)$, of order $n$ of $T$ is defined as follows:

$$
\Lambda_{n}(T):=\max \left\{\lambda_{n}(T ; x):-1 \leqslant x \leqslant 1\right\}
$$

where

$$
\lambda_{n}(T ; x):=\sum_{k=1}^{n}\left|e_{k}(T ; x)\right| \text { for all } x \in[-1,1]
$$

is the so-called Lebesgue function of order $n$ of $T$ and

$$
\begin{array}{r}
\ell_{k}(T ; x):=\frac{\left(x-x_{1}\right)\left(x-x_{2}\right) \ldots\left(x-x_{k-1}\right)\left(x-x_{k+1}\right) \ldots\left(x-x_{n}\right)}{\left(x_{k}-x_{1}\right)\left(x_{k}-x_{2}\right) \ldots\left(x_{k}-x_{k-1}\right)\left(x_{k}-x_{k+1}\right) \ldots\left(x_{k}-x_{n}\right)} \\
\text { for } k=1,2,3, \ldots, n
\end{array}
$$

are the fundomental polynomials of Lagronge interpolation based on the $n$th row of $T$; that is, on the nodes $x_{1}, x_{2}, x_{3}, \ldots, x_{n}$. Equivalently, $\Lambda_{n}(T)$ may be regarded as the norm of the Lagrange interpolation operator $L_{n}: \mathcal{C}[-1,1] \rightarrow C[-1,1]$, where

$$
\begin{aligned}
\left(L_{n}(f)\right)(x): & =L_{n}(f ; x) \\
& =\sum_{k=1}^{n} \mathrm{f}\left(\mathrm{x}_{\mathrm{k}}\right) \ell_{k}(x) .
\end{aligned}
$$

The Lebesgue constants play a fundamental rôle in interpolation theory, and estimates for the Lebesgue constants have been given since the early 1900 s.

Bernstein [2] obtained the following estimate, valid for all $n \in N$ :

$$
\Lambda_{n}(T)<8+\frac{4}{\pi} \log n \text {. }
$$

See ([16], pp. 48-50) for a proof of this result. In 1963, this result was improved by Bexman [1]:

$$
\Lambda_{n}(T)<4 \sqrt{2}+\frac{2}{\pi} \log n .
$$

Much earliex, in 1931, Berstein [3] had given the following asymptotic estimate for $\Lambda_{n}(T)$ :

$$
\Lambda_{n}(T) \sim \frac{2}{\pi} \log n \text {. }
$$

In 1965, Luttmann and Rivlin [13], in view of extensive numerical computations, conjectured that 


$$
\Lambda_{n}(T)=\sum_{k=1}^{n}\left|e_{k}(1)\right|
$$

and established the following asymptotic expression:

$$
\lim _{n \rightarrow \infty}\left[\Lambda_{n}(T)-\frac{2}{\pi} \log n\right]=\frac{2}{\pi}\left[\gamma+\log \frac{8}{\pi}\right]=0.9625 \ldots
$$

where $\gamma=0.5772 \ldots$ is Euler's constant.

Luttmann and Rivlin's conjecture was later proved by Ehlich and Zeller [6]; see also Powell ([18], p. 407) and Rivlin ([20], pp. 14-16).

Rivlin [19] also showed that the asymptotic tendency was monotone in character and obtained the estimate:

$$
\Lambda_{n}(T) \leqslant 1+\frac{2}{\pi} \log n \text { for } n=1,2,3, \ldots
$$

More recently, in 1980, Günttner [12] gave the following asymptotic estimate for $\Lambda_{n}(T)$ :

$$
\begin{aligned}
& \Lambda_{n}(T)=\frac{2}{\pi} \log n+\frac{2}{\pi}\left[\gamma+\log \frac{8}{\pi}\right]+\alpha_{n}, \text { where } \\
& 0<\alpha_{n}<\frac{\pi}{72 n^{2}} \text { for } n=1,2,3, \ldots
\end{aligned}
$$

In 1982, Shivakumar and Wong [23] established the complete asymptotic expansion for $\Lambda_{n}(T)$ :

$$
\left\{\begin{array}{l}
\Lambda_{n}(T) \sim \frac{2}{\pi} \log n+\frac{2}{\pi}\left[\gamma+\log \frac{8}{\pi}\right]+\frac{8}{\pi} \sum_{k=1}^{\infty} \frac{A_{k}}{n^{2 k}} \text {, where } \\
A_{k}:=\frac{(-1)^{k-1}\left(2^{2 k-1}-1\right)^{2}}{(2 k)(2 k) !}\left(\frac{\pi}{2}\right)^{2 k} B_{2 k}^{2} \text { for } k=1,2,3, \ldots \text { and } \\
B_{2 k} \text { for } k=1,2,3, \ldots \text { represent Bernoulli numbers. }
\end{array}\right.
$$

It should be noted that the above asymptotic expansion appeared in Giinttner's doctoral dissertation in 1972 ([11], p. 79).

We turn now to Hermite-Fejér interpolation. In this case, given a real-valued function $f$ on $[-1,1]$ and $n \in N$, there is a unique polynomial $H_{n}(f, T ; x)$ of degree not exceeding $2 n-1$ such that

$$
H_{n}\left(f, T ; x_{k}\right)=f\left(x_{k}\right) \text { for } k=1,2,3, \ldots, n
$$

and 


$$
H_{n}^{\prime}\left(f, T ; x_{k}\right)=0 \text { for } k=1,2,3, \ldots, n \text {, }
$$

where $x_{1} x_{2} x_{3} \ldots x_{n}$ is the $n$th row of the Chebyshev matrix $T$. $H_{n}(f, T ; x)$ is called the Hermite-Fejer interpolation polynomial of degree not exceeding $2 n-1$ associated with $f$ and based on the nodes $x_{1}, x_{2}, x_{3}, \ldots, x_{n}$; that is, on the $n$th row of $T$.

In 1916, Fejér [7] gave a constructive proof of the celebrated Weierstrass approximation theorem.

THEOREM 1. [Fejér] If $f \in \mathcal{C}[-1,1]$, then

$$
\lim _{n \rightarrow \infty}\left\|H_{n}(f, T)-f\right\|_{\infty}=0 \text {, }
$$

where $\|\cdot\|_{\infty}$ denotes the uniform norm on $C[-1,1]$.

The first uniform error estimate for the rate of convergence of Hermite-Fejér interpolation was provided by Popoviciu [17] in 1950. His estimate was framed in terms of the modulus of continuity of $f$, which is defined as follows:

$$
\omega(f ; \delta):=\sup \{|f(x)-f(y)|: x, y \in[-1,1],|x-y| \leqslant \delta\} .
$$

THEOREM 2. [Popoviciu] If $f \in \mathcal{C}[-1,1]$, then for $n=1,2,3, \ldots$,

$$
\left\|H_{n}(f, T)-f\right\|_{\infty} \leqslant 2 \omega\left(f ; \frac{1}{\sqrt{n}}\right) \text {. }
$$

This does not represent a very fast rate of convergence.

Popoviciu's result was subsequently improved by Moldovan [15] and later, from a different point of view, by Shisha and Mond [22].

THEOREM 3. [Moldovan; Shisha and Mond] If $f \in \mathcal{C}[-1,1]$, then for $n=4,5,6, \ldots$,

$$
\left\|H_{n}(f, T)-f\right\|_{\infty} \leqslant c_{1} \omega\left(f ; \frac{\log n}{n}\right),
$$

where $c_{1}$ is an absolute positive constant.

The next major improvement in estimating $\| H_{n}(f, T)-f_{\infty}$ came from Bojanic [4] in 1969. His estimate was framed in terms of the arithmetic means of the sequence $\left\{w\left(f ; \frac{1}{k}\right)\right\}_{k=1}^{n}$. 
Let $\Omega(\delta)$ be an increasing, subadditive, continuous function defined on $\mathrm{R}^{+}$with $\Omega(0)=0$. Given $M>0$, define:

$$
C_{M}(\Omega):=\{f \in C[-1,1]: \omega(f ; \delta) \leqslant M \Omega(\delta) \text { for all } \delta \geqslant 0\} \text {. }
$$

THEOREM 4. [Bojanic] There exist positive constants $c_{2}$ and $c_{3}$ such that for $n=2,3,4, \ldots$,

$$
\frac{c_{2} M}{n} \sum_{k=2}^{n} \Omega\left(\frac{1}{k}\right) \leqslant \sup \left\{\left\|H_{n}(f, T)-f\right\|_{\infty}: f \in \mathcal{C}_{M}(\Omega)\right\} \leqslant \frac{c_{3} M}{n} \sum_{k=1}^{n} \Omega\left(\frac{1}{k}\right) .
$$

The next refinements in upper estimates came from Vértesi [24] in 1971 and Saxena [21] in 1974.

THEOREM 5. [Vértesi; Saxena] There is a positive constant $c_{4}$ such that for $n=2,3,4, \ldots$ and $x \in[-1,1]$,

$$
\left|H_{n}(f, T ; x)-f(x)\right| \leqslant \frac{c_{4}}{n} \sum_{k=1}^{n} \omega\left(f ; \frac{\sqrt{1-x^{2}}}{k}+\frac{1}{k^{2}}\right) .
$$

Thus we see that the precision of approximation is considerably better at the endpoints than it may be at the centre of the interval. This is not surprising, for the zeros of $T_{n}(x)$ are themselves concentrated towards the endpoints of $[-1,1]$.

However, none of the previous estimates satisfies the condition that if $x=x_{k}$ is a node of interpolation, then both the error and the error estimate vanish simultaneously. In other words, none of the previous estimates reflects the interpolation conditions.

But an estimate which does was given by De Vore $([5]$, p. 44) in 1972. THEOREM 6. [De Vore] If $f \in \mathcal{C}[-1,1]$, then for $n=1,2,3, \ldots$ and $x \in[-1,1]$,

$$
\left|H_{n}(f, T ; x)-f(x)\right| \leqslant 2 \omega\left(f ; \frac{\left|T_{n}(x)\right|}{\sqrt{n}}\right) .
$$

If $T_{n}(x)=0$, then $H_{n}(f, T ; x)=f(x)$ and so the pointwise error estimate in (15) reflects the interpolation conditions. However this estimate is not precise when $x$ is not a node of interpolation.

The next improvement was obtained by Goodenough and Mills [10] in 1981. 
THEOREM 7. [Goodenough and Mills] There exist positive constants $c_{5}$ and $c_{6}$ such that, if $f \in C[-1,1], n=2,3,4, \ldots$ and $x \in[-1,1]$. $\left|H_{n}(f, T ; x)-f(x)\right| \leqslant \frac{c_{5}}{n} T_{n}(x){ }^{2} \sum_{k=1}^{n} \omega\left(f ; \frac{\sqrt{1-x^{2}}}{k}+\frac{1}{k^{2}}\right)+c_{6} \omega\left(f ; \frac{\left|T_{n}(x)\right|}{n}\right)$.

As far as asymptotic estimates for Hermite-Fejér interpolation are concerned, we shall consider the maximum interpolation error, $\Delta_{n}(T)$, when a Lipschitz class of functions is approximated by Hermite-Fejer interpolation polynomials. More precisely, denote by Lip 1 the class of all Lipschitz 1 functions on $[-1,1]$, where

$$
f \in \operatorname{Lip} 1 \Longleftrightarrow|f(x)-f(y)| \leqslant|x-y| \text { for all } x, y \in[-1,1] \text {. }
$$

Put

$$
\Delta_{n}(T ; x):=\sup \left\{\left|H_{n}(f, T ; x)-f(x)\right|: f \in \operatorname{Lip} 1\right\} \text { for all } x \in[-1,1]
$$

and define

$$
\Delta_{n}(T):=\max \left\{\Delta_{n}(T ; x):-1 \leqslant x \leqslant 1\right\} .
$$

We call $\Delta_{n}(T)$ the maximum interpolation error for functions of class Lip 1 by Hermite-Fejér interpolation polynomials of degree not exceeding $2 n-1$.

Theorem 4 can be modified to show that $\Delta_{n}(T)$ is asymptotically like $\frac{\log n}{n}$. This result was subsequently improved by Mills ([14], p. 114) in 1980 , who showed that:

$$
\Delta_{n}(T)=\frac{2}{\pi} \frac{\log n}{n}+0\left(\frac{1}{n}\right), \text { as } n \rightarrow \infty .
$$

In other words, $\frac{2}{\pi} \frac{\log n}{n}$ is the dominant term in the complete asymptotic expansion for $\Delta_{n}(T)$.

In 1985, Goodenough [8] gave the complete asymptotic expansion for $\Delta_{n}(T):$

$$
\left\{\begin{array}{l}
\Delta_{n}(T) \sim \frac{2}{\pi} \frac{\log n}{n}+\frac{2}{\pi}\left[\log \frac{8}{\pi}+\gamma\right] \frac{1}{n}+4 \sum_{k=1}^{\infty} \frac{A_{k}^{*}}{n^{2 k+1}} \text { as } n=2 m \rightarrow \infty, \\
\text { where } A_{k}^{*}=\frac{(-1)^{k-1}\left(2^{2 k-1}-1\right)^{2} B_{2 k}^{2}}{2 k(2 k) !}\left(\frac{\pi}{2}\right)^{2 k-1} \text { for } k=1,2,3, \ldots .
\end{array}\right.
$$


If we now compare the complete asymptotic expansion for $\Lambda_{n}(T)$ given by (12) with the complete asymptotic expansion for $n \Delta_{n}(T)$, then we find that they are identical. This is a totally unexpected result, for $\Lambda_{n}(T)$ and $\Delta_{n}(T)$ arise from fundamentally different problem areas.

The aim of this paper is to establish the following close link between Lebesgue constants and Hermite-Fejêr interpolation:

THEOREM 8. For even values of $n$,

$$
\Lambda_{n}(T)=n \Delta_{n}(T) \text {. }
$$

\section{Technical Preliminaries}

We first need explicit formulations for $\Lambda_{n}(T)$ and $\Delta_{n}(T)$. Using the fact that $\lambda_{n}(T ; x)$ attains its maximum value at $x= \pm 1$, Ehlich and Zellex [6] have proved that

$$
\Lambda_{n}(T)=\frac{1}{n} \sum_{k=1}^{n} \cot \frac{(2 k-1) \pi}{4 n} .
$$

A proof of this identity also appears in ([20], pp. 14-17).

we next recall that the formula for $H_{n}(f, T ; x)$ is given by:

$$
H_{n}(f, T ; x)=\sum_{k=1}^{n} f\left(x_{k}\right) h_{k}(x) \text {, }
$$

where

$$
h_{k}(x)=\frac{1}{n^{2}}\left(1-x x_{k}\right)\left(\frac{T_{n}(x)}{x-x_{k}}\right)^{2}
$$

and

$$
x_{k}=\cos \frac{(2 k-1) \pi}{2 n} \text { for } k=1,2,3, \ldots, n \text {. }
$$

From (13), (14) and the uniqueness of $H_{n}(f, T ; x)$ we have

$$
H_{n}(1, T ; x)=\sum_{k=1}^{n} h_{k}(x) \equiv 1 \text {. }
$$

For $-1 \leqslant x \leqslant 1$, define 


$$
\phi_{x}(t):=|x-t| \text { for all } t \in[-1,1]
$$

on the one hand, $\phi_{x} \in$ Lip 1 and hence

$$
\Delta_{n}(T ; x) \geqslant\left|H_{n}\left(\phi_{x}, T ; x\right)-\phi_{x}(x)\right|=\sum_{k=1}^{n}\left|x-x_{k}\right| h_{k}(x) .
$$

on the other hand, for any $f \in \operatorname{Lip} 1$, we have by (21) and (23)

$$
\left|H_{n}(f, T ; x)-f(x)\right|=\left|\sum_{k=1}^{n}\left[f\left(x_{k}\right)-f(x)\right] h_{k}(x)\right| \leqslant \sum_{k=1}^{n}\left|x-x_{k}\right| h_{k}(x) .
$$

It follows that

$$
\Delta_{n}(T ; x)=\sum_{k=1}^{n}\left|x-x_{k}\right| h_{k}(x)
$$

Goodenough [9] has proved that, for even values of $n, \Delta_{n}(T ; x)$ attains its maximum value at $x=0$. It follows that

$$
\begin{aligned}
\Delta_{n}(T) & :=\max \left\{\Delta_{n}(T ; x):-1 \leqslant x \leqslant 1\right\} \\
& =\max \left\{\sum_{k=1}^{n}\left|x-x_{k}\right| h_{k}(x):-1 \leqslant x \leqslant 1\right. \\
& =\frac{2}{n^{2}} \sum_{k=1}^{m} \frac{1}{x_{k}} \text { for } n=2 m \text { even. }
\end{aligned}
$$

Now put $x=\cos \theta$. Then for even values of $n$,

$$
\begin{aligned}
\Delta_{n}(T) & =\frac{2}{n^{2}} \sum_{k=1}^{m} \sec \frac{(2 k-1) \pi}{2 n} \\
& =\frac{2}{n^{2}} \sum_{k=1}^{m} \operatorname{cosec} \frac{(2 k-1) \pi}{2 n} .
\end{aligned}
$$

It follows that

$$
n \Delta_{n}(T)=\frac{2}{n} \sum_{k=1}^{m} \operatorname{cosec} \frac{(2 k-1) \pi}{2 n}
$$

Formulae (20) and (25) provide the cornerstone for the proof of Theorem 8 . 


\section{Proof of Theorem 8.}

In view of (20) and (25), it will suffice to show that

$$
\begin{aligned}
\cot \frac{(2 k-1) \pi}{4 n}+\cot \frac{(2(n+1-k)-1) \pi}{4 n}= & 2 \operatorname{cosec} \frac{(2 k-1) \pi}{2 n} \\
& \text { for } k=1,2,3, \ldots, m .
\end{aligned}
$$

Now

$$
\begin{aligned}
\cot & \frac{(2 k-1) \pi}{4 n}+\cot \frac{(2(n+1-k)-1) \pi}{4 n} \\
& =\frac{\sin \frac{(2 k-1) \pi}{4 n} \cos \frac{(2 n-2 k+1) \pi}{4 n}+\cos \frac{(2 k-1) \pi}{4 n} \sin \frac{(2 n-2 k+1) \pi}{4 n}}{\sin \frac{(2 k-1) \pi}{4 n} \sin \frac{(2 n-2 k+1) \pi}{4 n}} \\
& =\frac{\sin \frac{\pi}{2}}{\sin \frac{(2 k-1) \pi}{4 n} \sin \frac{(2 n-2 k+1) \pi}{4 n}} \\
& =\frac{2}{2 \sin \frac{(2 k-1) \pi}{4 n} \cos \frac{(2 k-1) \pi}{4 n}} \\
& =2 \operatorname{cosec} \frac{(2 k-1) \pi}{2 n} \text { for } k=1,2,3, \ldots, m .
\end{aligned}
$$

This concludes the proof of Theorem 8 .

REMARKS. The function $\Delta_{n}(T ; x)$ defined by (16) plays an analogous rôle to that played by the Lebesgue function $\lambda_{n}(T ; x)$ in the development of results concerning Lebesgue constants. Computer graphs reveal that, for even values of $n$, the local maxima of $\Delta_{n}(T ; x)$ are monotonically increasing as we proceed from either of the endpoints of $[-1,1]$ to the centre of the interval. A proof of this result has yet to be given. Contrast this phenomenon with the behaviour of the Lebesque function, whose local maxima are monotonically decreasing as we proceed from either of the endpoints of $[-1,1]$ to the centre of the interval.

Furthermore, the asymptotic expansion for $\Delta_{n}(T)$ for odd values of $n$ has yet to be given. An inherent difficulty in the solution of this problem is that, for odd values of $n=2 m+1$, the global maximum of $\Delta_{n}(T ; x)$ is located slightly to the left of the midpoint of $\left[x_{m+2}, x_{m+1}\right]$. 


\section{References}

[1] D.L. Berman, "On the best grid system for parabolic interpolation" (Russian), Iav. Vyss. Ucebn. Zaved. Matematika 4 (1963), 20-25.

[2] S.N. Bernstein, "Quelques remarques sur 1'interpolation", Commanic. Math. Assoc. Kharkov 15 (1916), 49-61.

[3] S.N. Bernstein, "Sur la limitation des valeurs d'une polynôme $P(x)$ de degré $n$ sur tout un segment par ses valeurs en $(n+1)$ points du segment" Izv. Akad. Nauk SSSR 7 (1931), 1025-1050.

[4] R. Bojanic, "A note on the precision of interpolation by HermiteFejér polynomials", Proceedings of the Conference on constructive theory of functions, Budapest 1969, 69-76 (Akadémiai Kiadó, Budapest, 1972).

[5] R.A. De Vore, The approximation of continuous functions by positive Zinear operators (Lecture Notes in Mathematics 293. SpringerVerlag, Berlin, Heidelberg, New York, 1972).

[6] H. Ehlich and $K$. Zeller, "Auswertung der Normen von Interpolationsoperatoren", Math. Ann. 164 (1966), 105-112.

[7] L. Fejêr, "Ueber Interpolation", Nachr. Akad. Wiss. Göttingen Math.Phys. KI. (1916), 66-91.

[8] S.J. Goodenough, "The complete asymptotic expansion for the degree of approximation of Lipschitz functions by Hermite-Fejer interpolation polynomials" $J$. Approx. Theory 44 (1985), 325-342.

[9] S.J. Goodenough, Error estimates for the approximation of functions by certain interpolation polynomials (Ph.D. thesis, University of Newcastle, 1985).

[10] S.J. Goodenough and T.M. Mills, "A new estimate for the approximation of functions by Hermite-Fejér interpolation polynomials", $J$. Approx. Theory 31 (1981), 253-260.

[11] R. Günttner, Abschätzrongen für Normen von Interpolationsoperatoren (Doctoral dissertation, Technical University of Clausthal, west Germany, 1972).

[12] R. Günttner, "Evaluation of Lebesgue constants", SIAM J. Numer. Anal. $17(1980), 512-520$. 
[13] F.W. Luttmann and T.J. Rivlin, "Some numerical experiments in the theory of polynomial interpolation", IBM J. Res. Develop. 2 (1965), 187-191.

[14] T.M. Mills, "Some techniques in approximation theory", Math. Scientist 5 (1980), 105-120.

[15] E. Moldovan, "Observatii asupra unor procedee de interpolare generalizate", Acad. Repub. Pop. Rom. Bul. Stinte Sect. Stinte Mat. Eiz. 6 (1954), 477-482.

[16] I.P. Natanson, Constructive function theory, Vol. III (Frederick Ungar, New York, 1965).

[17] T. Popoviciu, "Asupra demonstratiei teoremai lui Weierstrass cu ajutorul polynoamelor de interpolare", Acad. Repub. Pop. Rom., Lucrarile sessiunii generala s,tintifice din 2-12 iunie, 1950 (1951), 1664-1667.

[18] M.J.D. Powell, "On the maximum errors of polynomial approximations defined by interpolation and by least squares criteria", Comput. J. 9 (1967), 404-407.

[19] T.J. Rivlin, "The Lebesgue constants for polynomial interpolation", Functional Analysis and its Applications, Int. Conf. Madrass 1973, 422-437 (Springer-Verlag, Berlin, 1974).

[20] T.J. Rivlin, The Chebyshev polynomials (Wiley-Interscience, New York, London, Sydney, Toronto, 1974).

[21] R.B. Saxena, "A note on the rate of convergence of Hermite-Fejer interpolation polynomials", Canad. Math. BuzZ. 17 (1974), 299-301.

[22] 0 . Shisha and $B$. Mond, "The rapidity of convergence of the HermiteFejer approximation to functions of one or several variables", Proc. Amer. Math. Soc. 16 (1965), 1269-1276.

[23] P.N. Shivakumar and R. Wong, "Asymptotic expansion of the Lebesgue constants associated with polynomial interpolation", Math. Comp. 39 (1982), 195-200. 
[24] P.O.H. Vértesi, "On the convergence of Hermite-Fejêr interpolation", Acta Math. Acad. Sci. Hungar. 22 (1971), 151-158.

Department of Mathematics, Statistics, and Computer science,

University of Newcastle,

N.S.W. 2308. 